\title{
An extinction of the conductive electrons of fullerene nano whisker as the air exposure effects
}

\author{
Tatsuya Doi, Kyouhei Koyama, Nobuyuki Aoki, Jonathan P. Bird*, \\ and Yuichi Ochiai \\ Graduate School of Advanced Integration Science, Chiba University, Chiba, Japan \\ Fax: 81-43-290-3427, E-mail: doitatuya@graduate.chiba-u.jp \\ *Department of Electrical Engineering, SUNY University at Buffalo, USA
}

\begin{abstract}
ESR spectra of fullerene nano-whiskers (FNW) are investigated to elucidate their electronic properties and the influence of oxygen absorption. A singlet ESR spectrum appears together with temperature independent Pauli-para type behavior in the area intensity (which indicates the magnetic susceptibility $\left(\chi^{\mathrm{ESR}}\right)$ ) in vacuum. Motional narrowing of the absorption width is observed in vacuum due to fast molecule motion. Absorption of oxygen eliminates Pauli-para behavior and motional narrowing. Furthermore, creation of localized spins is observed and $\chi^{\mathrm{ESR}}$ shows no Pauli-para contribution after absorption. A kind of metal insulator transition due to gas absorption is therefore observed by ESR in the system of $\mathrm{C}_{60} \mathrm{FNW}$.
\end{abstract}

Key words: fullerene nano whisker, $\mathrm{C}_{60}$, organic semiconductor, $\mathrm{ESR}$, oxygen

\section{INTRODUCTION}

Recently, organic semiconductor devices have been developed as field effect transistors (FET) [1], electro luminescence (EL) devices [2] and photo voltaic cells (PVC) [3]. In such applications, both $n$ - and $p$-type semiconductors are required to achieve the desired device functionality. $\mathrm{C}_{60}$ fullerene has been widely studied because it exhibits the highest mobility among $n$-type organic semiconductors. For example, much research has focused on using fullerene materials for high mobility FETs and photo devices. Generally, the electronic properties of organic materials are easily affected by their environment, and have many vacancies in their crystal structure. Consequently, gas molecules, such as oxygen, nitrogen, and water vapor, are easily absorbed into the solid, and can cause large changes of the electronic properties. Usually, absorption of oxygen molecules prevents $n$-type organic semiconductors from being utilized for device applications in ambient air, due to the creation of charged trap states.

Electron spin resonance (ESR) has previously been applied to elucidate the behavior of the conduction electrons in organic materials. ESR is a powerful and sensitive method for detecting electron spins and characterizing their electrical properties. Examples include the observation of metallic behavior in the fullerene Langmuir-Blodgett films due to the alkali-metal doping [4], and studies of gate-voltage induced carriers in pentacene FETs [5] and polythiophene [6] FETs.

In recent years, new types of $\mathrm{C}_{60}$ crystals have been fabricated by Miyazawa and coworkers, using the liquid-liquid interfacial precipitation method (LLIP) to form crystalline fullerene nano-whiskers (FNW) [7]. These FNW have been used as the channel of organic FETs [8] and as a material for PVC [9]. At the same time, some interesting features, such as the solvent dependence of the crystal structure [10], and structural modification due to evaporation of the crystal solvent [11], have also been reported. As a matter of course, such characteristics should inevitably affect the electronic property of FNW, although the details of this connection remain to be clarified.

In this paper, we discuss the electronic structure of FNW under both ambient and vacuum conditions. In particular, we describe how air absorption leads to a reduction in the number of conduction electrons in these nanostructures. These results will be important for the development of FNWs with characteristics that are stable under air exposure, and so should lead to the development of new functional FNWs for application to electronics. At the end of the paper, we mention the necessity of performing polymerization of FNW to allow their ambient applications.

\section{EXPERIMENT}

2.1 Fabrication of fullerene nano-whisker

FNWs were synthesized by use of the LLIP method [7]. $\mathrm{A} \mathrm{C}_{60}(>99.95 \%$, MTR) saturated $m$-xylene $(>98.0 \%$, Kanto Kagaku) solution was prepared, and filtered through a fine membrane $(0.25-\mu \mathrm{m}$ pore size) to remove residual $\mathrm{C}_{60}$ and other indissoluble matter (e.g. as oxidized fullerene). The resulting solution was then disembogued into a glass vial, following which isopropyl alcohol (IPA, $>99.9 \%$, Kanto Kagaku) was disembogued onto the solution to form the liquid-liquid interface. The solvent ratio $\left(\mathrm{C}_{60}\right.$ saturated $m$-xylene solution:IPA) was $1: 4$, and this solution was stored in a refrigerator at $280 \mathrm{~K}$ for a few weeks. Subsequently, the synthesized FNW were filtered with a paper filter, then dried and put into a quartz tube for X-band ESR measurements. The amount of the dried sample is $0.11 \mathrm{~g}$ which was estimated after ESR measurement. The morphology [12] and quality [13] of the FNW is known to depend importantly upon the conditions of the LLIP method. 


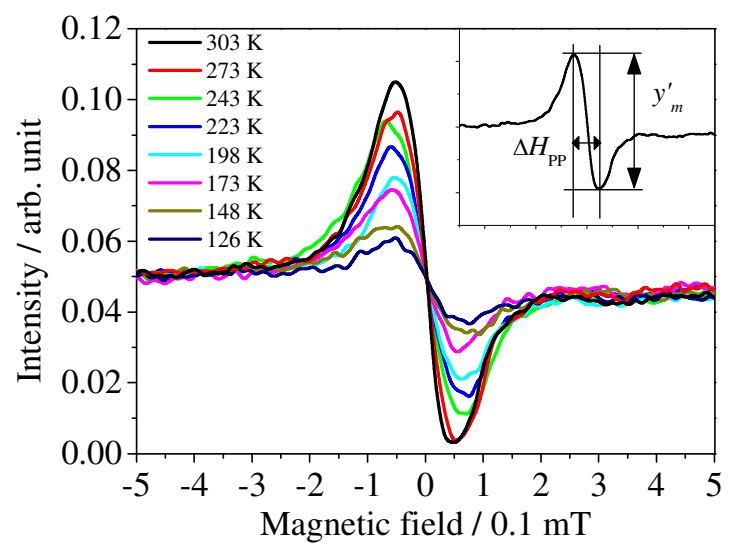

Fig. 1. ESR spectra of the FNW measured at different temperatures in vacuum.

\subsection{Electron spin resonance measurements}

ESR measurement was carried out using a conventional X-band ESR spectrometer, together with a temperature-control system that operated between 120 and $423 \mathrm{~K}$. Samples were annealed for 15 hours at 423 $\mathrm{K}$ in vacuum $\left(\sim 4 \times 10^{-4} \mathrm{~Pa}\right)$ before ESR measurement. This annealing process has also been utilized in prior studies of nanowhisker-based FET devices [8], to remove solvent and gas molecules.

\section{RESULTS AND DISCUSSION}

3.1 ESR measurements in vacuum

ESR spectra of the FNW were obtained at various temperatures after annealing. The ESR technique is usually applied to materials which have unpaired electrons and/or conductive electrons. Due to its molecular structure, a single molecule of $\mathrm{C}_{60}$ has no unpaired or conductive electrons. This property has also been reported for $\mathrm{C}_{60}$ solution [14]. However, Maniwa et al. have reported an ESR signal in sublimed $\mathrm{C}_{60}$ solid [15]. It was also observed that the paramagnetic spins were removed by repeating the sublimation so that the spins might originate from some kind of paramagnetic impurities. In Ref. [15], the measured ESR intensity was clearly found to decrease on air exposure, and similar effects have also been observed in ESR of nano-graphites [16]. On the other hand, in the case of ESR in such graphites, it is thought that the edge localized spins of graphite are affected by the paramagnetism of oxygen molecules, leading to a degradation of the ESR intensity [17]. These two results indicate a good accordance between paramagnetic impurities in solid $\mathrm{C}_{60}$ and edge-localized spins in nanographites.

With regards to electron transport, the excitation energy of solid $\mathrm{C}_{60}$ is reported to be $0.22 \mathrm{eV}$ under vacuum conditions [18]. This indicates that $\mathrm{C}_{60}$ thin films have shallow trap states, with activation energy of $0.22 \mathrm{eV}$ in vacuum. In such an electron system, electrons occupying the trap states can behave as conductive electrons at room temperature. A finite number of conduction electrons should therefore appear, allowing a conduction electron resonance to be observed in the $\mathrm{C}_{60}$ solid.

ESR spectra under vacuum are shown in Fig. 1. The parameters $y_{\mathrm{m}}^{\prime}$ and $\Delta H_{\mathrm{PP}}$ defined in the inset are the

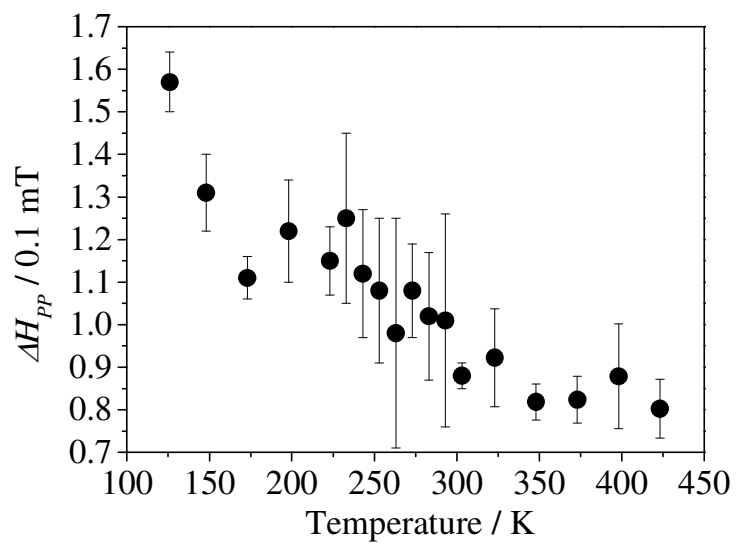

Fig 2. Temperature dependence of $\Delta H_{\mathrm{PP}}$ of FNW in vacuum.

intensity and peak-to-peak width of the ESR spectrum, respectively. These spectra were obtained for a microwave power of $1 \mathrm{~mW}$. The intensity of the ESR increases with increasing temperature, and broadening of $\Delta H_{\mathrm{PP}}$ is observed at low temperatures. The temperature dependence of $\Delta H_{\mathrm{PP}}$ is shown in Fig. 2 and indicates that $\Delta H_{\mathrm{PP}}$ is reduced with increasing temperature. The $\Delta H_{\mathrm{PP}}$ indicates an anomaly around 230 $\mathrm{K}$. As well known, phase transition from face-centered cubic structure (fcc) to simple cubic structure (sc) is observed at $260 \mathrm{~K}$ in $\mathrm{C}_{60}$ crystal [19]. Lower shift of transition temperature can be caused by weak interaction between molecules and molecule interaction in FNW should be weaker than usual fcc $\mathrm{C}_{60}$ crystal due to lager lattice constant [20]. Therefore, the anomaly of $\Delta H_{\mathrm{PP}}$ should be related to the phase transition.

$\Delta H_{\mathrm{PP}}$ is given by Eq. 1 [14] and $T_{2}$ can be estimated to be $4.10 \times 10^{-8} \mathrm{~s}$ at room temperature. We propose that these results indicate motional narrowing, because the $T_{2}$ at room temperature is sufficiently long in comparison with the motional correlation time of $\mathrm{C}_{60}$, reported to be $9.1 \mathrm{ps}$ at $283 \mathrm{~K}$ [21]. Such motional narrowing has never been reported in ESR of solid $\mathrm{C}_{60}$. To observe motional narrowing, two requirements must be satisfied: (1) the molecular correlation time should be shorter than $T_{2}$, and; (2) the spins should follow the molecular motion. Therefore, the spins should follow the $\mathrm{C}_{60}$ molecular motion as the molecules freely rotate in vacuum. However, the estimated value of $T_{2}$ was affected by motional narrowing as discussed before; that is to say, local magnetic field of the FNW is homogenized by molecule motion. Therefore, the spins should interact with much inhomogeneous local field without molecule motion. Such inhomogeneity of local field causes faster relaxation. Consequently, the original $\Delta H_{\mathrm{PP}}$ can be considered from Fig. 2 and speculated to be broader than $0.16 \mathrm{mT}$ at low temperature.

$$
\begin{gathered}
\Delta H_{P P}=\frac{h}{2 \sqrt{3} \pi g \mu_{B} T_{2}} \\
\chi^{E S R} \propto N_{S} \propto S \propto \Delta H_{P P}^{2} \cdot y_{m}^{\prime}
\end{gathered}
$$

Figure 3 shows the ESR magnetic susceptibility $\left(\chi^{\mathrm{ESR}}\right)$ as a function of $1 / T \cdot \chi^{\mathrm{ESR}}$ can be calculated from 


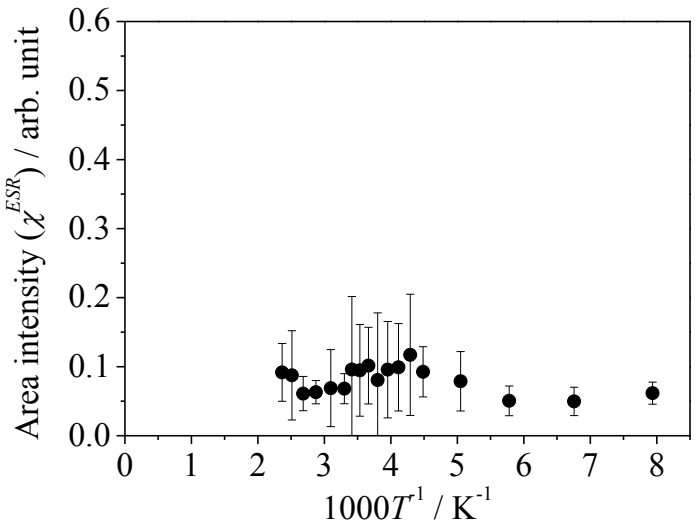

Fig 3. Temperature dependence of the magnetic susceptibility of FNWs in vacuum.

Eq.2 (Ref. 22), in which $S$ and $N_{\mathrm{S}}$ are the area intensity of the ESR spectrum and the spin number respectively. In Fig. $3, \chi^{\mathrm{ESR}}$ indicates a temperature independent Pauli-para type behavior in vacuum. From our results we conclude that the FNW show metallic electron properties, consistent with prior studies of their electrical properties. Specifically, Ogawa and coworkers demonstrated that FNW-FETs have a low on/off ratio, around 100 , because of gap states caused by structural disorder in the FNW [23]. As grown FNW usually show a hexagonal (hcp) structure, however this converts into a face-centered cubic (fcc) structure after evaporation of the solvent. This results in a high density of dislocations due to the change of crystal structure [24]. In our experiments, ESR measurements were carried out after high-temperature annealing. Therefore, almost all solvent molecules should be removed, leading to many structural defects in the FNW crystal. These defects should generate shallow localized states near the Fermi level. In fact, generation of spins in $\mathrm{C}_{60}$ crystals has been observed at the glass transition point, around $90 \mathrm{~K}$, due to the appearance of inter-gap levels [25]. Temperature independent $\chi^{\mathrm{ESR}}$ has also been observed in nanographites, and explained in terms of contributions from conduction electrons and edge-localized spins [16].

\subsection{Air exposure and ESR in ambient atmosphere}

After the ESR measurements in vacuum, ambient atmosphere was introduced into the quartz sample tube. ESR spectra obtained after this are shown in Fig. 4. The change of the area intensity is indicated in the inset of Fig. 4. The area intensity is increased 2.4 times after air exposure. Following this, we observed a recovery of the original area intensity after re-vacuuming. These variations are also summarized in the inset of Fig. 4. Since the spin number $\left(N_{\mathrm{S}}\right)$ and the area intensity $(S)$ should be proportional to each other, we conclude that absorption of air causes a $2.4 \times$ increase of $N_{\mathrm{S}}$. This result clearly shows that absorption of air, most likely oxygen, creates many spins in FNW. Also, the recovery of the original $S$ value means that absorbed oxygen can easily be removed simply by use of vacuuming. Therefore, the key phenomenon associated with the absorption can be considered to be a physical absorption, because the induced change shows reversibility. In other words, irreversible chemical absorption does not occur.

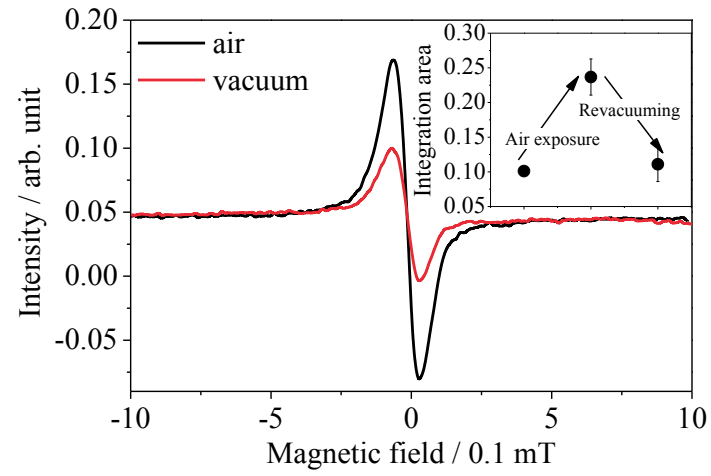

Fig. 4. ESR spectrum in vacuum (red line) and in air (black line).

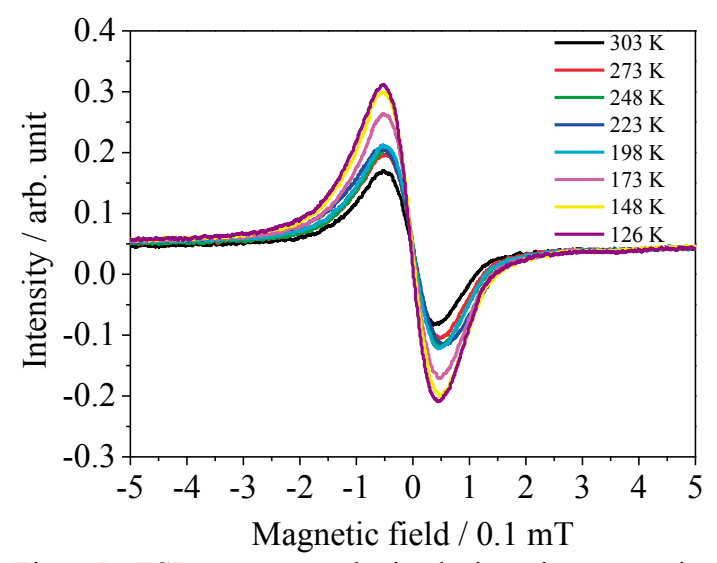

Fig. 5 ESR spectra obtained in air at various temperatures.

ESR spectra in air are shown in Fig. 5 at various temperatures and indicate that the intensity decreases with increase of temperature. The behavior can associated with the usual temperature dependence of paramagnetic spins. This behavior is the opposite of that discussed already for ESR in vacuum (Fig. 1). A temperature-independent feature of $\Delta H_{\mathrm{PP}}$ was observed in air as shown in Fig. 6, indicating the absence of motional narrowing. Belahmer et al. reported a hindrance of molecular rotation due to intercalation of oxygen molecules in solid $\mathrm{C}_{60}$ [26]. This mechanism partially explains our ESR result. It is necessary that the rotational correlation time should be three orders of magnitude larger to explain the temperature independent $\Delta H_{\text {Pp. }}$. However, in the case of hindrance of molecular motion, $\Delta H_{\mathrm{PP}}$ should reach broader than $0.16 \mathrm{mT}$ because the condition might be considered as same to low temperature in vacuum. However, $0.11 \mathrm{mT}$ of $\Delta H_{\mathrm{PP}}$ is a maximum value in observation as shown in Fig. 6. This disagreement between $\Delta H_{\mathrm{PP}}$ under air and vacuum might illustrate creation of new states. Also, a pronounced decrease of $T_{2}$ can cause a temperature-independent $\Delta H_{\mathrm{PP}}$, even if the molecules are rotating with a short molecular correlation time. However, in this case, it is required that $T_{2}$ decrease to become similar to the motional correlation time (9.1 ps [21]). Such a reduction requires $\Delta H_{\mathrm{PP}}$ increase to up to $360 \mathrm{mT}$. Therefore, we propose that the conduction electrons might exist at trap states which are not affected 


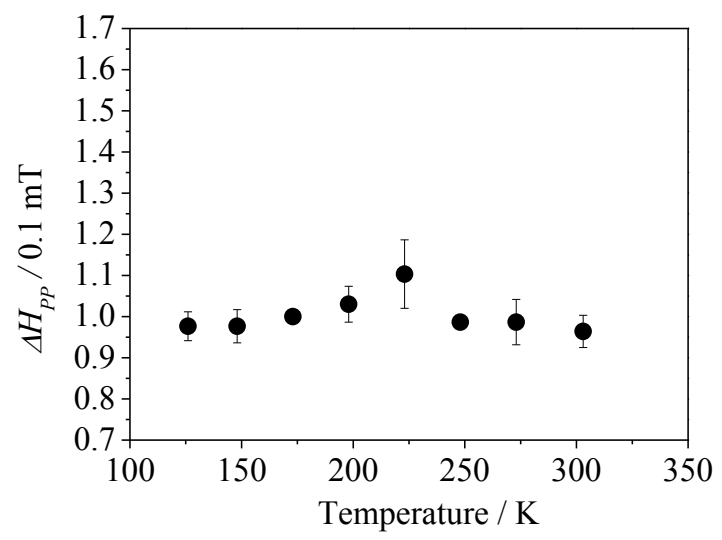

Fig 6 Temperature dependence of $\Delta H_{\mathrm{PP}}$ of FNWs in vacuum.

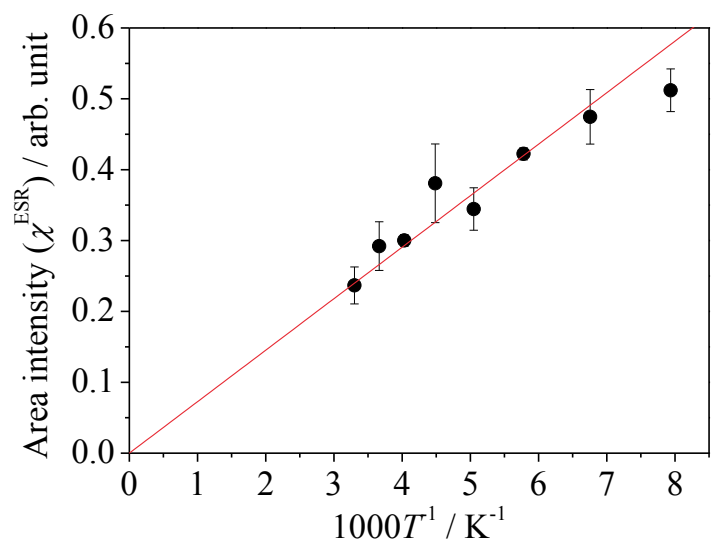

Fig 7. Temperature dependence of magnetic susceptibility of FNWs in air (close circle). Guide line shows Curie law (red line).

by molecule motion and/or huge prolonging of molecule correlation time might cause both of temperature independent $\Delta H_{\mathrm{PP}}$ and destruction of gap states. Nevertheless, the extended spin states over $\mathrm{C}_{60}$ molecule has been strongly affected and disturbed by air absorption.

A clear proportional dependence of $\chi^{\mathrm{ESR}}$ on $T$ was obtained after air exposure indicating a Curie law (Fig. 7). Typical paramagnetic spin that exhibit such behavior include lattice imperfections and/or free radicals, both of which are isolated spins. It has been reported that $\mathrm{O}_{2}$ molecules create deep trap states through absorption into the $\mathrm{C}_{60}$ crystal [18]. The resulting state is localized, and is located $0.55 \mathrm{eV}$ below the conduction band [18]. This state can behave as a trap for charge carriers and may create isolated paramagnetic spins that are, at the same time, non-conducting. The role of $\mathrm{O}_{2}$-induced trap states is also suggested by the increase of $N_{\mathrm{S}}$ due to air absorption, as discussed already.

Our results indicated the observed ESR in FNW shows conductive property in vacuum and non-conductive property in air. This result clearly explains low on/off operation of FNW-FET in vacuum and difficulty to operation in air.

\section{CONCLUSIONS}

Electronic properties of FNW have been reported by

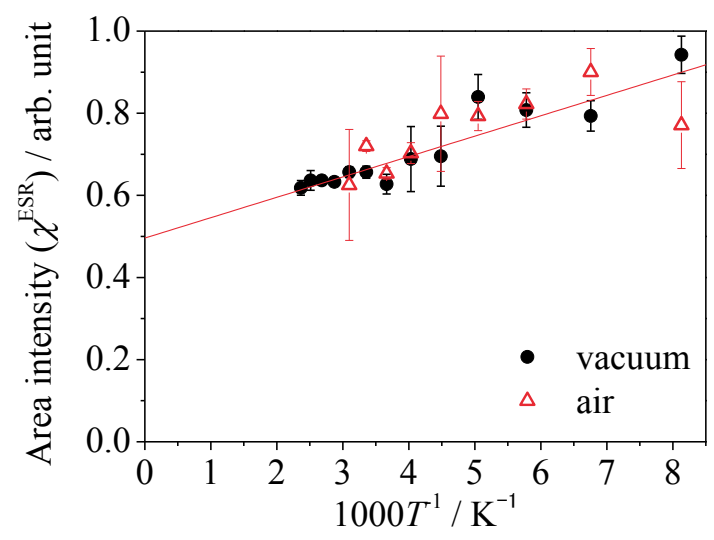

Fig. 8. Temperature dependence of magnetic susceptibilities which are assigned by ESR absorption component in $\mathrm{C}_{60}$ powder sample by using Eq. 2 .

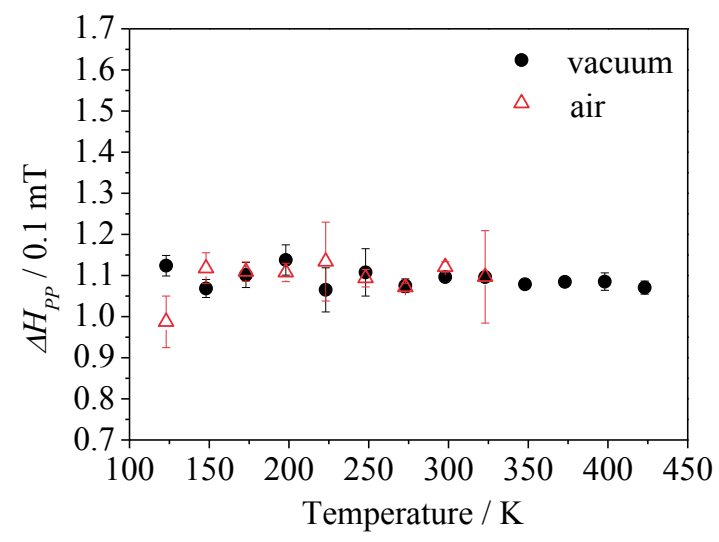

Fig. 9. Temperature dependence of $\Delta H_{\mathrm{PP}}$ of ESR absorption component in $\mathrm{C}_{60}$ powder

ESR under vacuum and air. We represented Pauli-para magnetic susceptibility of the FNW in vacuum, which indicates FNW has a metallic feature. Clear atmosphere absorption effect of FNW has been observed with a disappearance of motional narrowing and Pauli-para feature. Absorption of oxygen molecules should strongly affect to electronic property of FNW, and the metallic feature has been destroyed. We presented that air absorption causes creation of localized electrons. We propose a kind of metal insulator transition caused by air absorption, furthermore that crystal modification such as polymerization will be needed to obtain air tolerable FNW.

\section{APPENDIX}

5.1 ESR spectrum of $\mathrm{C}_{60}$ powder

To discuss the features in the ESR of FNW, we should clarify the ESR spectrum of $\mathrm{C}_{60}$ powder samples. It is well known that ESR-active centers have been observed in $\mathrm{C}_{60}$. However, a clear temperature dependence has not been perfectly reported.

The $\mathrm{C}_{60}$ power sample was systemized by MTR Ltd. and was of $99.95 \%$ purity. In the case of $\mathrm{FNW}$, the $\mathrm{C}_{60}$ saturated $m$-xylene was filtered before crystal growth. Therefore, all insoluble matter, such as oxidized fullerene and graphite-like carbon, were removed from the solution, as in previous reports [14], where the authors concluded that the origin of the ESR signal of 
$\mathrm{C}_{60}$ is the oxidized fullerene.

In our observation, $0.125 \mathrm{~g}$ of $\mathrm{C}_{60}$ powder was put into the ESR sample tube. Before the measurement, annealing was performed at $\sim 5 \times 10^{-4} \mathrm{~Pa}$ at $421 \mathrm{~K}$ for 22 h. Following this, we measured the temperature dependence of the ESR spectrum of the $\mathrm{C}_{60}$ powder in vacuum and in air. In vacuum, the $\mathrm{C}_{60}$ powder showed a temperature dependent magnetic susceptibility (Fig. 8). The results illustrate that $\mathrm{C}_{60}$ powder sample includes paramagnetic spins originating from oxidized fullerene, as discussed in Ref. 14. According to the air exposure effect of FNW, such a Curie type behavior should appear only in ESR performed in air. Also, the ESR spectrum is not affected by air exposure in the case of $\mathrm{C}_{60}$ powder sample. The above two behaviors show clearly that the ESR spectrum of $\mathrm{C}_{60}$ powder sample originates from radical species. We also measured the temperature dependence of $\Delta H_{\mathrm{PP}}$. Both in air and under vacuum conditions, this $\Delta H_{\mathrm{PP}}$ was clearly temperature independent (Fig.9.). However, in the case of FNW, as we discussed above, $\Delta H_{\mathrm{PP}}$ shows motional narrowing with increasing temperature. These experimental results indicate that the molecular motion of FNW is faster than for $\mathrm{C}_{60}$ powder. It must be that substitutional group of the oxidized $\mathrm{C}_{60}$ prevents rotational motion of the $\mathrm{C}_{60}$ molecules.

In conclusion, our observations clearly show that the ESR spectrum of FNW is inherently different from that of $\mathrm{C}_{60}$ powder, so that ESR observation gives clear information on electron transport in FNW.

\section{ACKNOWLEDGMENTS}

This work is supported in part by Grants-in-Aid for Scientific Research from Japan Society for the Promotion of Science (19054016, 19204030 and $16656007)$ and by JSPS Core-to-Core Program. This work was also in part supported by Global COE program at Chiba University (G-03, MEXT) and promoted by international research and educational collaboration between Chiba University and SUNY Buffalo.

\section{REFERENCES}

[1] A. L. Briseno, S. C. B. Mannsfeld, M. M. Ling, S. Liu, R. J. Tseng, C. Reese, M. E. Roberts, Y. Yang, F. Wudl and, Z. Bao, Nature, 444, 913-917 (2006)

[2] P. Matyba, K. Maturova, M. Kemerink, N. D. Robinson and, L. Edman, Nature Materials, 8, 672-676 (2009)

[3] P. Peumans, S. Uchida and, S. R. Forrest, Nature, 425, 158-162 (2003)

[4] K. Ikegami, S. Kuroda, M. Matsumoto and, T. Nakamura, Japanese Journal of Applied Physics, 34, L1227-L1229 (1995)

[5] K. Marumoto, S. Kuroda, T. Takenobu and, Y. Iwasa Physical Review Letters, 97, 256603 1-256603 4 (2006) [6] H. Tanaka, S. Watanabe, H. Ito, K. Marumoto and, S. Kuroda, Applied Physics Letters, 94, 103008 1-103008 3 (2009)

[7] K. Miyazawa, Y. Kuwasaki, A. Obayashi and, M. Kuwabara, Journal of Materials Research, 17, 83-88 (2002)

[8] K. Ogawa, T. Kato, A. Ikegami, H. Tsuji, N. Aoki and, Y. Ochiai, Applied Physics Letters, 88, 112109 1-112109 3 (2006)

[9] P. R. Somani, S. P. Somani and, M. Umeno, Applied Physics Letters, 91, 173503 1-173503 3 (2007)

[10] K. Miyazawa, J. Minato, T. Yoshii, M. Fujino, and T. Suga, Journal of Materials Research, 20, 688-695 (2005)

[11] J. Minato and, K. Miyazawa, Diamond \& Related Materials, 15, 1151-1154 (2006)

[12] K. Hotta and, K. Miyazawa, Journal of Physics: Conference Series, 159, 012021 1-012021 4 (2009)

[13] K. Miyazawa and, K. Hotta, Journal of Crystal Growth, 312, 2764-2770 (2010)

[14] Arturo Calligiani and, Carlo Taliani, Chemical materials, 6, 1633-1637 (1994)

[15] Y. Maniwa, M. Naagasaka, A. Ohi, K. Kume, K. Kikuchi, K. Saito, I. Ikemoto, S. Suzuki and, Y. Achiba, Japanese Journal of the Applied Physics, 33, L173-L176 (1993)

[16] V. Yu. Osipov, A. I. Shames, T. Enoki, K. Takai, M. Endo, T. Hayashi, Y. Kaburagi and, A. Ya. Vul', Diamond \& Related Materials, 18, 220-223 (2009)

[17] V. Yu. Osipov, A. I. Shames, T. Enoki, K. Takai, M. Endo, Y. Kaburagi and, A. Ya. Vul', Diamond \& Related Materials, 19, 492-495 (2010)

[18] H. Habuchi, S. Nitta, D. Han and, S. Nonomura, Journal of Applied Physics, 87, 8580-8588 (2000)

[19] R. Tycko, G. Dabbagh, R. M. Fleming, R. C. Haddon, A. V. Makhija and, S. M. Zahurak, Physical Review Letters, 67, 1886-1889 (1991)

[ 20 ] J. Minato and, K. Miyazawa, Carbon, 43, 2837-2841 (2005)

[21] R. D. Johnson, C. S. Yannoni, H. C. Dorn, J. R. Salem and, D. S. Bethune, Science, 255, 1235-1238 (1992)

[22 ] M. Havlicek, W. Jantsch, M. Rummeli, R. Schonfelder, K, Yanagi, Y, Miyata, H. Kataura, F. Simon, H. Peterlik and, H. Kuzmany, Physical Status Solidi B, 247, 2851-2854 (2010)

[23] K. Ogawa, N. Aoki, K. Miyazawa, S. Nakamura, T Mashino, J. P. Bird and, Y. Ochiai, Japanese Journal of Applied Physics, 47, 501-504 (2008)

[24] K. Miyazawa, J. Minato, T. Yoshii, M. Fujino and, T. Suga, Journal of Material Research, 20, 688-695 (2005)

[25] Y. Ishijima and, T. Ishiguro, Journal of Physical Society of Japan, 66, 2948-2949 (1997)

[26] Z. Belahmer, P. Bernier, L. Firlej, J. M. Lambert and, M. Tibet, Physical Review B, 47, 15980-15983 (1993)

(Received December 27, 2010; Accepted August 8, 2011) 